\title{
A SIMULATION STUDY TO ASSESS THE EFFICACY OF LINEAR CONTROL THEORY MODELS FOR THE COORDINATION OF A TWO-STAGE CUSTOMIZED SERVICE SUPPLY CHAIN
}

\author{
Douglas J. Morrice \\ Edward G. Anderson \\ McCombs School of Business \\ Management Department \\ The University of Texas at Austin \\ 1 University Station B6300 \\ Austin, TX 78712, U.S.A.
}

\author{
Saurav Bharadwaj \\ Manufacturing and Decision Systems Engineering \\ The University of Texas at Austin \\ 1 University Station C2200 \\ Austin, TX 78712, U.S.A.
}

\begin{abstract}
In this paper, we conduct a simulation study to evaluate linear control theory models applied to the management and coordination of a two-stage customized service supply chain. Linear models that were proposed in previous research are compared against more general nonlinear models for three different levels of coordination: centralized, decentralized, and no information sharing. Using simulation and regression analysis, we show that the linear models yield results that are off by an average of six percent or less for parameter values observed in practice.
\end{abstract}

\section{INTRODUCTION}

Over the past fifteen years there has been a great deal of work, both industrial and academic, devoted to improving supply chain management through better information sharing and improved coordination of supply chain activities. This paper focuses on the evaluation of models used to assess different levels of coordination and information sharing in two-stage customized service supply chains. Examples of such supply chains are found in oilfield services; outsourced engineering and technical services; consulting; financial services such as mortgages and insurance; commercial construction; mobile workforces such as home health care providers; and even make-to-order manufacturers such as capital equipment producers.

Figure 1 provides an example from oilfield services. The first stage, called evaluation, involves exploration to find and characterize an oil and gas reservoir. At the second stage, the reservoir is developed for production. While Figure 1 provides a very high level view an oilfield services supply chain, it is a perspective around which the major companies involved in oilfield services such as Schlumberger (<www. schlumberger. com>), Halliburton (<www . halliburton.com $>$ ), and Baker-Hughes ( $<$ www . bak erhughes. com $>$ ) organize their businesses.

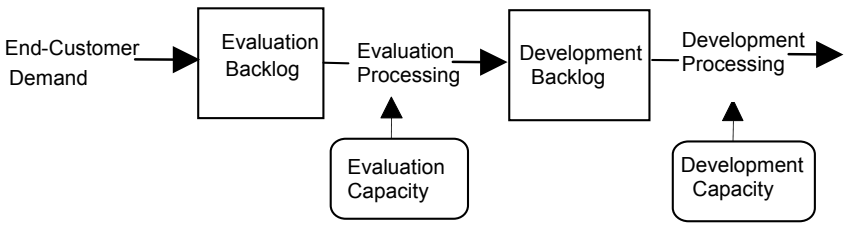

Figure 1: A High Level View of an Oilfield Services Supply Chain

Anderson and Morrice (2000, 2002) and Anderson et al. (2004a, 2004b) analyze coordination and information sharing in customized services supply chains using simulation and control theory. In particular, Anderson et al. (2004a) formulate and solve optimal linear control problems that approximate more general optimal non-linear control problems of centralized, decentralized, and no information sharing management strategies in a two-stage customized service supply chain. In this paper, we assess these linear approximations relative to their non-linear counterparts using simulation. We show that the linear approximations can perform quite well especially in light of the model parameter settings in practice across many different industries.

It is important to note that simulation and control theory have been used to analyze manufacturing supply chains. Towill $(1995,1996)$ use simulation and filter theory to study supply chain dynamics. Dejonckheere et al. (2003) proposes a control theoretic approach to measure and avoid the bullwhip effect (i.e., order quantity variance amplification at progressively higher stages of the supply chain away from the original demand signal). Perea-Lopez et al. $(2001,2003)$ use dynamic modeling and a model predictive control strategy for performance improvement and optimization of a supply network. They compare the performance of the supply network under centralized and decentralized management approaches. Kempf (2004) pro- 
poses a model predictive control approach to supply chain management in semiconductor manufacturing.

Section 2 describes the control problem results from Anderson et al. (2004a). Section 3 contains the simulation results. In Section 4, we discuss the simulation results in light of empirical data available on several industries.

\section{THE CONTROL PROBLEMS}

Let the index $t$ represent time. To formulate optimal control problems for the supply chain in Figure 1, Anderson et al. (2004a) assume that end customer demand, $r(t)$, is Gaussian with mean $\bar{r}$ and standard deviation $\sigma_{r}$. The Gaussian demand is used because it is assumed that there is a great deal of aggregation in the demand at the supply chain level. Further, for $i=1,2$, let

$C_{i}(t)=$ the capacity at stage $i$ at time $t$. The productivity of $C_{i}(t)$ is assumed to be unity without loss of generality. $P_{i}(t)=$ the processing rate at stage $i$ at time $t$. Note, $P_{i}(t) \leq \lambda_{i} C_{i}(t)$ for all $t \geq 0$ and $P_{0}(t)=r(t)$. $W_{i}(t)=$ the net rate of change in capacity (or net hiring) at

stage $i$ at time $t$. This is the control variable at stage $i$.

$B_{i}(t)=$ the backlog and work-in-progress at stage $i$ at time $t$. $\tilde{B}_{i}=$ the target backlog and work-in-progress at stage $i$.

$\varphi_{i}^{2}=$ the per unit cost of excess or insufficient backlog at stage $i$.

$\psi_{i}^{2}=$ the per unit cost of changing capacity at stage $i$.

$v_{i}=$ the fraction of jobs at stage $i$ that proceed to the next stage.

$\lambda_{i}=$ the average fraction of capacity that can be utilized in production at stage $i$.

Assume $W_{i}(t)$ is continuous for $i=1,2$ and $t \geq 0$. Given the initial backlogs $B_{i}(0)$ and capacities $C_{i}(0)$ for $i=1,2$ at time $t=0$, a closed-loop optimal control problem for centralized control of the two stages simultaneously is, for $t \geq 0$,

$$
\begin{aligned}
& Z=\min _{W_{1}(t), W_{2}(t)} \lim _{T \rightarrow \infty} \\
& {\left[\sum_{i=1}^{2} E\left[\frac{1}{T} \int_{0}^{T}\left\{\begin{array}{l}
\phi_{i}^{2}\left[B_{i}(t)-\tilde{B}_{i}\right]^{2} \\
+\psi_{i}^{2}\left[W_{i}(t)\right]^{2}
\end{array}\right\} d t\right]\right)}
\end{aligned}
$$

subject to

$$
\begin{aligned}
& \frac{d B_{i}(t)}{d t}=v_{i-1} P_{i-1}(t)-P_{i}(t), \text { for } \mathrm{i}=1,2 \\
& \lambda_{i} C_{i}(t) \quad \text { if } B_{i}(t)>0 \\
& P_{i}(t)=\left\{\min \left(v_{i-1} P_{i-1}(t), \lambda_{i} C_{i}(t)\right) \quad\right. \text { otherwise } \\
& \text { for } i=1,2 \\
& \frac{d C_{i}(t)}{d t}=W_{i}(t), \text { for } \mathrm{i}=1,2 \\
& B_{i}(t), C_{i}(t) \geq 0 \text {, for } \mathrm{i}=1,2 \text {. }
\end{aligned}
$$

By "closed-loop" we indicate that the determination of the value of all control variables $W_{i}(t)$ will incorporate the values of $B_{i}(t), C_{i}(t)$, and all $r(u)$ for $0 \leq u \leq t$, where for $i=1,2$ and $t \geq 0$.

For the decentralized control problem, each stage optimizes its own objective function subject to its local constraints on backlog and change in capacity. Therefore, under the same assumptions, a decentralized closed loop control problem for stage $i(i=1,2)$ and for $t \geq 0$, is

$$
\begin{aligned}
& Z_{i}=\min _{W_{i}(t)} \lim _{T \rightarrow \infty} \\
& E\left[\frac{1}{T} \int_{0}^{T}\left\{\begin{array}{l}
\phi_{i}^{2}\left[B_{i}(t)-\tilde{B}_{i}\right]^{2} \\
+\psi_{i}^{2}\left[W_{i}(t)\right]^{2}
\end{array}\right] d t\right]
\end{aligned}
$$

subject to

$$
\begin{aligned}
& \frac{d B_{i}(t)}{d t}=v_{i-1} P_{i-1}(t)-P_{i}(t) \\
& \lambda_{i} C_{i}(t) \quad \text { if } B_{i}(t)>0 \\
& P_{i}(t)=\left\{\min \left(v_{i-1} P_{i-1}(t), \lambda_{i} C_{i}(t)\right) \quad\right. \text { otherwise } \\
& \frac{d C_{i}(t)}{d t}=W_{i}(t) \\
& B_{i}(t), C_{i}(t) \geq 0 \text {. }
\end{aligned}
$$

The objective functions in (1) and (6) balance variation in backlog and net hiring about targets. The former is a measure of service quality (consistency) and the latter is a measure of resource management. Equations (2) and (7) are balance of flow constraints on the change in backlog. Expressions (3) and (8) limit a stage's processing rate by its capacity or what flows in from a previous stage. Constraints (4) and (9) define the control variables. Finally, (5) and (10) are non-negativity constraints.

In order to generate approximate solutions to the optimal control problems, Anderson et al (2004a) make the following simplifying assumption: the target backlogs and work in are high enough that backlog rarely, if ever, drops to zero. Hence, processing is always determined by capacity. Under this assumption, the centralized optimal control problem can be approximated by the following linearly relaxed formulation for $t \geq 0$ :

$$
\begin{aligned}
& Z=\min _{W_{1}(t), W_{2}(t)} \lim _{T \rightarrow \infty} \\
& {\left[\sum_{i=1}^{2} E\left[\frac{1}{T} \int_{0}^{T}\left\{\begin{array}{l}
\phi_{i}^{2}\left[B_{i}(t)-\tilde{B}_{i}\right]^{2} \\
+\psi_{i}^{2}\left[W_{i}(t)\right]^{2}
\end{array}\right] d t\right]\right)}
\end{aligned}
$$

subject to 


$$
\begin{gathered}
\frac{d B_{i}(t)}{d t}=v_{i-1} \lambda_{i-1} C_{i-1}(t)-C_{i}(t), \text { for } \mathrm{i}=1,2 \\
\frac{d C_{i}(t)}{d t}=W_{i}(t), \text { for } \mathrm{i}=1,2 .
\end{gathered}
$$

where $C_{0}(t)=r(t)$. Similarly, for stage for stage $i(i=1,2)$ and for $t \geq 0$, the decentralized control problem can be approximated by linear relaxation model:

$$
\begin{aligned}
& Z_{i}=\min _{W_{i}(t)} \lim _{T \rightarrow \infty} \\
& E\left[\frac{1}{T} \int_{0}^{T}\left\{\begin{array}{l}
\phi_{i}^{2}\left[B_{i}(t)-\tilde{B}_{i}\right]^{2} \\
+\psi_{i}^{2}\left[W_{i}(t)\right]^{2}
\end{array}\right] d t\right]
\end{aligned}
$$

subject to

$$
\begin{gathered}
\frac{d B_{i}(t)}{d t}=v_{i-1} \lambda_{i-1} C_{i-1}(t)-C_{i}(t) \\
\frac{d C_{i}(t)}{d t}=W_{i}(t) .
\end{gathered}
$$

Note that the survival rates $v_{i}$ and the fraction of productive capacity $\lambda_{i}$ will have no dynamic effect on the models if the costs and productivities at the each stage are scaled appropriately. Hence, without loss of generality, we will set all $v_{i}$ and $\lambda_{i}$ equal to unity and suppress them to simplify exposition.

The linearly relaxed control problems can be solved to yield control rules that are functions of the backlog and capacity. In particular, the control rules for the centralized control problem are of the form:

$$
\begin{aligned}
& W_{1}(t)=Q_{C 1}^{(1)}\left[C_{1}(t)-\bar{r}\right]+Q_{C 2}^{(1)}\left[C_{2}(t)-C_{1}(t)\right] \\
& +Q_{B 1}^{(1)}\left[B_{1}(t)-\tilde{B}_{1}\right]+Q_{B 2}^{(1)}\left[B_{2}(t)-\tilde{B}_{2}\right], \\
& W_{2}(t)=Q_{C 1}^{(2)}\left[C_{1}(t)-\bar{r}\right]+Q_{C 2}^{(2)}\left[C_{2}(t)-C_{1}(t)\right] \\
& +Q_{B 1}^{(2)}\left[B_{1}(t)-\tilde{B}_{1}\right]+Q_{B 2}^{(2)}\left[B_{2}(t)-\tilde{B}_{2}\right]
\end{aligned}
$$

where the coefficients are (complicated) functions of the $\varphi_{i}^{2}$ and the $\psi_{i}^{2}$ and have the following (intuitive) signs

$$
\begin{aligned}
& Q_{C 1}^{(1)}<0, Q_{C 2}^{(1)}>0, Q_{B 1}^{(1)}>0, Q_{B 2}^{(1)}<0, \\
& Q_{C 1}^{(2)}<0, Q_{C 2}^{(2)}<0, Q_{B 1}^{(2)}>0, Q_{B 2}^{(2)}>0
\end{aligned} .
$$

The superscripts on the coefficients refer to the stage of the control rule. The subscripts denote whether or not the coefficient is associated with a term that contains capacity or backlog and at what stage. To illustrate, $Q_{C 2}^{(1)}$ is the coefficient in the first stage control rule associated with second stage capacity. With everything else fixed, if second stage capacity is higher than the first stage capacity (i.e., $\left.C_{2}(t)>C_{1}(t)\right)$, then net hiring $W_{1}(t)$ will be positive at the first stage (because $\left.Q_{C 2}^{(1)}>0\right)$ in order to move $C_{1}(t)$ in the direction of $C_{2}(t)$ and bring the two back into balance. As another example, consider the coefficient in the second stage control rule associated with the first stage backlog, i.e., $Q_{B 1}^{(2)}$. If the backlog at the first stage exceeds it target, then (with everything else fixed), net hiring at the second stage will increase (because $Q_{B 1}^{(2)}>0$ ) in anticipation of an increase of demand that will eventually flow through to the second stage. For the purposes of this paper, we do not provide the complete expressions for the coefficients because they are large and complicated functions of $\varphi_{i}^{2}$ and the $\psi_{i}^{2}$. The interested reader is referred to Anderson et al. (2004).

Similarly, the control rules for the decentralized problem are

$$
\begin{aligned}
& \widehat{W}_{1}(t)=\widehat{Q}_{C 1}^{(1)}\left[C_{1}(t)-\bar{r}\right]+\widehat{Q}_{C 2}^{(1)}\left[C_{2}(t)-C_{1}(t)\right] \\
& +\widehat{Q}_{B 1}^{(1)}\left[B_{1}(t)-\tilde{B}_{1}\right]+\widehat{Q}_{B 2}^{(1)}\left[B_{2}(t)-\tilde{B}_{2}\right], \\
& \widehat{W}_{2}(t)=\widehat{Q}_{C 1}^{(2)}\left[C_{1}(t)-\bar{r}\right]+\widehat{Q}_{C 2}^{(2)}\left[C_{2}(t)-C_{1}(t)\right] \\
& +\widehat{Q}_{B 1}^{(2)}\left[B_{1}(t)-\tilde{B}_{1}\right]+\widehat{Q}_{B 2}^{(2)}\left[B_{2}(t)-\tilde{B}_{2}\right]
\end{aligned}
$$

where the coefficients are (complicated) functions of the $\varphi_{i}^{2}$ and the $\psi_{i}^{2}$ and have the following (intuitive) signs

$$
\begin{aligned}
& \widehat{Q}_{C 1}^{(1)}<0, \widehat{Q}_{C 2}^{(1)}=0, \widehat{Q}_{B 1}^{(1)}>0, \widehat{Q}_{B 2}^{(1)}=0, \\
& \widehat{Q}_{C 1}^{(2)}<0, \widehat{Q}_{C 2}^{(2)}<0, \widehat{Q}_{B 1}^{(2)}>0, \widehat{Q}_{B 2}^{(2)}>0
\end{aligned} .
$$

Note that $\widehat{Q}_{C 2}^{(1)}=0$ and $\widehat{Q}_{B 2}^{(1)}=0$ because in the decentralized model, stage 1 optimizes it own stage myopically and is not influenced by stage 2's backlog or capacity. On the other hand, even though stage 2 optimizes its own stage myopically, it is assumed to have knowledge of stage 1's backlog and capacity because the output for stage 1 is the input for stage 2 . In order to reflect the reality found in many supply chains, Anderson et al. (2004a) propose a third non-optimal control rule in which it is assumed that stage 1's backlog is not known to stage 2 (stage 1 capacity can always be inferred at stage 2 because it is equivalent to stage 2 demand). This control rule is a simple modification of (22) in which $\widehat{Q}_{B 1}^{(2)}$ is set equal to zero. This third control rule is referred to as the "no backlog information" or simply "no information" case.

The remainder of this paper is devoted to evaluating the efficacy of the linear relaxation models for the centralized, decentralized, and no information cases using simulation.

\section{SIMULATION RESULTS}

Anderson et al. (2004a) provide a preliminary simulation analysis in which they simulate the results of the general 
closed loop control problems in (1) - (5) and (6) - (10) using the linear optimal control rules for centralized, decentralized, and no information sharing cases and compare them against linear relaxation model results described in Section 2. The comparison is based on $Z$ (i.e., the objective or penalty function for the entire supply chain). We extend that simulation analysis to a full two-level factorial design and formally analyze it using regression analysis (Box and Draper 1987).

The experimental design depends on the factors $\varphi_{1}, \varphi_{2}, \psi_{1}, \psi_{2}$ because, except for demand variance $\sigma_{r}$, these factors completely characterize the solutions to the control problems. We do not include $\sigma_{r}$ in the experimental design because it appears is a scaling factor in $Z$. This was verified mathematically in the linear relaxation models and via simulation for the more general models. Hence, $\sigma_{r}$ is set to one. Without loss of generality, $\varphi_{1}$ is also set equal can be set equal to one and the other factors are defined as relative costs in order to simplify the experimental design by one dimension. In order to simplify the mathematical analysis in Anderson et al. (2004a), the factor

$\delta=\left(\frac{\psi_{2}}{\psi_{1}}\right) \quad$ is used in place of $\psi_{2}$. Hence we will use $\delta$ in place of $\psi_{2}$ as well. The high and low values for $\psi_{1}, \delta$, and $\phi_{2}$ are 2 and 0.5 , respectively. These values were chosen to represent relative costs that are two times (or four times when the parameters are squared) the other relative costs in any combination.

Two other factors are used in the experimental design: a common target backlog $\tilde{B}$ and a common target capacity $\tilde{C}$ for both stages. Common targets suffice because results for the linear relaxation models are expected to degrade as $\tilde{B}$ and $\tilde{C}$ decrease regardless of the stage because constraints such as (3) and (5) or (8) and (10) are more likely to be violated. Since average demand and supply must match in this model, $\tilde{C}$ determines the average level of demand so the latter does not need to be considered as a separate factor in the design.

The high and low values for $\tilde{B}$ and $\tilde{C}$ are 1.645 and 1.96 respectively. Assuming Gaussian white noise demand, backlog and capacity would hit zero 5 percent and 2.5 percent of the time breaking the linearly relaxation assumption that neither of these fall to zero. Hence, we believe that this is a region over which the assumptions of the linear relaxation models might be stressed. From other pilot simulation runs, we found that when target values are above 1.96, the results from the linear relaxation models correspond very closely to the results from the general models.

We perform a separate regression analysis for centralized, decentralized, and no information cases. The regression response is the percentage by which the simulation results for a general model differ from the analytical results from a linear relaxation model. The experimental design contains 32 points ( 5 factors at 2 levels). At each design point, 30 simulations of 1000 periods are made. The 1000 time periods are chosen based on visual inspection of the simulation sample paths (similar to Welch's procedure see Law and Kelton 2000) to ensure convergence of the $Z$. The average of the percentage differences from the 30 simulation runs is used as the regression response in an attempt to satisfy the regression assumption of normally distributed random error.

The regression results for the three cases are shown in Tables 1, 2 and 3. They were generated using a stepwise regression procedure implemented in StatPro (Albright et al. 2003) in which all main and two-way interactions effects are considered for entry into the model. At each step, a variable with $\mathrm{p}$-value for the $\mathrm{t}$-value (i.e., the t-statistic for a regression coefficient) of less than 0.05 enters and any variables with $p$-values for the $t$-values greater than 0.10 exit from the model.

The three models fit the data very well (as measured by $R^{2}$ and Adjusted $R^{2}$ statistics). Additionally, the four main regression assumptions or normality, linearity, homoscedasticitiy, and independence were found to hold (i.e., none were rejected) using standard utilities in Statpro for testing these assumptions (see Albright et al. 2003).

All these models yield remarkably similar results. In all cases, increasing target backlog (denoted by T_Backlog) or target capacity (denoted by T_Capacity) decreases the difference between the general and linear relaxation models. Additionally, a very slight reinforcing interaction effect exists between $\tilde{B}$ and $\tilde{C}$. These results for $\tilde{B}$ and $\tilde{C}$ are expected because the farther the targets are from zero, the less chance there is of backlog and capacity falling to zero violating the main assumption of the linear relaxation models. Target backlog turns out to be the most significant factor, having regression coefficients that are roughly an order of magnitude greater than target capacity coefficients. In other words, a low backlog target (or a greater possibility of the backlog falling to zero) is more detrimental to the performance of the linear relaxation models than the low capacity target level. In addition, target backlog has coefficients with magnitudes that are at least twice the size of the coefficients associated with the next most significant factor.

Increasing per unit capacity costs $\left(\psi_{1}, \delta\right)$ increases the average of the differences between the general model and the linearly relaxed model. This correlates with the sensitivity of the results to the backlog. If the capacity costs are high, then variations in the demand are absorbed by the backlog making the backlog more likely to hit zero. An analogous explanation exists for the fact that increasing per unit backlog costs $\left(\phi_{2}\right)$ decreases the average of the differences between the general model and the linearly relaxed model.

All other interaction effect terms are relatively small and less intuitive. Hence, their impact on the regression results will not be discussed for the sake of brevity.

It is important to note that these regressions results confirm that with a target backlog of 1.96 percent, the linear relaxations models yield results that, on average, are within about six percent of the results generated by the 
Table 1: Regression Results for the Average of the Percentage Difference between General Model Simulation Results and the Linear Relation Model Analytical Results for the Centralized Case

\begin{tabular}{|l|r|r|r|r|}
\hline Summary measures & & & & \\
\hline Multiple R & 0.9997 & & & \\
\hline R-Square & 0.9994 & & & \\
\hline Adjusted R-Square & 0.9991 & & & \\
\hline StErr of Est & 0.0008 & & & \\
\hline & & & & \\
\hline Regression coefficients & & & & \\
\hline & Coefficient & Std Err & \multicolumn{1}{c|}{ t-value } & p-value \\
\hline Constant & 0.0908 & 0.0001 & 616.5891 & 0.0000 \\
\hline T_Backlog & -0.0266 & 0.0001 & -180.8077 & 0.0000 \\
\hline Psi1 & 0.0066 & 0.0001 & 44.9900 & 0.0000 \\
\hline Delta & 0.0045 & 0.0001 & 30.7225 & 0.0000 \\
\hline Phi2 & -0.0022 & 0.0001 & -14.7420 & 0.0000 \\
\hline Phi2*Delta & -0.0018 & 0.0001 & -12.5199 & 0.0000 \\
\hline T_Capacity & -0.0016 & 0.0001 & -10.8214 & 0.0000 \\
\hline T_Capacity*Phi2 & 0.0007 & 0.0001 & 5.0278 & 0.0001 \\
\hline T_Capacity*Delta & -0.0007 & 0.0001 & -4.6290 & 0.0001 \\
\hline T_Backlog*Delta & 0.0005 & 0.0001 & 3.2497 & 0.0038 \\
\hline T_Capacity*T_Backlog & -0.0004 & 0.0001 & -2.4013 & 0.0257 \\
\hline
\end{tabular}

Tab1e 2: Regression Results for the Average of the Percentage Difference between General Model Simulation Results and the Linear Relation Model Analytical Results for the Decentralized Case

\begin{tabular}{|l|r|r|r|r|}
\hline Summary measures & & & & \\
\hline Multiple R & 0.9985 & & & \\
\hline R-Square & 0.9971 & & & \\
\hline Adjusted R-Square & 0.9957 & & & \\
\hline StErr of Est & 0.0019 & & & \\
\hline & & & & \\
\hline Regression coefficients & & & & \\
\hline & Coefficient & Std Err & t-value & p-value \\
\hline Constant & 0.0884 & 0.0003 & 261.9282 & 0.0000 \\
\hline T_Backlog & -0.0252 & 0.0003 & -74.6733 & 0.0000 \\
\hline Psi1 & 0.0076 & 0.0003 & 22.5677 & 0.0000 \\
\hline Phi2 & -0.0074 & 0.0003 & -21.9403 & 0.0000 \\
\hline Phi2*Delta & -0.0057 & 0.0003 & -16.9538 & 0.0000 \\
\hline Delta & 0.0046 & 0.0003 & 13.5698 & 0.0000 \\
\hline T_Capacity & -0.0034 & 0.0003 & -10.1317 & 0.0000 \\
\hline T_Capacity*Delta & -0.0022 & 0.0003 & -6.4980 & 0.0000 \\
\hline T_Backlog*Delta & 0.0015 & 0.0003 & 4.4829 & 0.0002 \\
\hline T_Capacity*T_Backlog & -0.0009 & 0.0003 & -2.5477 & 0.0187 \\
\hline Phi2*Psi1 & 0.0008 & 0.0003 & 2.4310 & 0.0241 \\
\hline
\end{tabular}


Tab1e 3: Regression Results for the Average of the Percentage Difference between General Model Simulation Results and the Linear Relation Model Analytical Results for the No Information Case

\begin{tabular}{|l|r|r|r|r|}
\hline Summary measures & & & & \\
\hline Multiple R & 0.9981 & & & \\
\hline R-Square & 0.9961 & & & \\
\hline Adjusted R-Square & 0.9946 & & & \\
\hline StErr of Est & 0.0024 & & & \\
\hline & & & & \\
\hline Regression coefficients & & & & \\
\hline & Coefficient & Std Err & t-value & p-value \\
\hline Constant & 0.0986 & 0.0004 & 236.3290 & 0.0000 \\
\hline T_Backlog & -0.0259 & 0.0004 & -61.9909 & 0.0000 \\
\hline Delta & 0.0134 & 0.0004 & 32.0451 & 0.0000 \\
\hline Psi1 & 0.0087 & 0.0004 & 20.8641 & 0.0000 \\
\hline Phi2 & -0.0054 & 0.0004 & -12.8982 & 0.0000 \\
\hline T_Capacity & -0.0048 & 0.0004 & -11.5965 & 0.0000 \\
\hline T_Capacity*Delta & -0.0028 & 0.0004 & -6.7895 & 0.0000 \\
\hline Phi2*Psi1 & 0.0016 & 0.0004 & 3.8862 & 0.0008 \\
\hline Phi2*Delta & -0.0014 & 0.0004 & -3.3063 & 0.0032 \\
\hline T_Capacity*T_Backlog & -0.0012 & 0.0004 & -2.8572 & 0.0092 \\
\hline
\end{tabular}

general optimal control models when the linear optimal control rules are used. Additionally, Anderson et al. (2004a) conduct relative comparisons of the centralized, decentralized, and no information cases using the results of the linear relaxation models. Since the regression models in this paper are so remarkably similar across all three cases (results degrade from the linear relaxation model in a similar fashion), their relative comparisons are robust even in the part of the nonlinear region that we have explored.

\section{DISCUSSION}

The simulation results indicate performance of the linear relaxation results are most sensitive to the assumption of positive backlog. They also indicate that the linear relaxation model objective functions are only about six percent lower than the general model objective functions when the target backlog is only 1.96 standard deviations from zero. So how does this square with reality? In other words, do average backlogs tend to be higher than this target in practices making the linear relaxation models viable predictors of reality.

We conducted an empirical study of 11 service industries. Days of backlog data were gathered from the Compustat Industrial Annual Database (Compustat Industrial Annual Data 2004). The results are presented in Table 4. The first column lists the industry designation. The sec ond lists the number of companies for which there is more than five years of annual data (the lower bound of five was chosen to get a reasonable estimate of the mean and stan-
Table 4: Industry Averages of the Number of Standard Deviations Average Days of Backlog is Away From Zero

\begin{tabular}{|l|c|c|c|}
\hline$\underline{\text { Industry }}$ & $\begin{array}{c}\# \text { of } \\
\text { Companies }\end{array}$ & $\underline{\underline{\text { Industry }}}$ & $\underline{\underline{\text { Standard }}}$ \\
\hline Business Services & 6 & 2.75 & $\underline{\underline{\text { Error }}}$ \\
\hline Construction & 12 & 4.05 & 0.26 \\
\hline $\begin{array}{l}\text { Services to } \\
\text { Dwellings }\end{array}$ & 1 & 3.28 & - \\
\hline $\begin{array}{l}\text { Management } \\
\text { Services }\end{array}$ & 3 & 3.67 & 1.57 \\
\hline $\begin{array}{l}\text { Computer Integrated } \\
\text { System Design }\end{array}$ & 66 & 2.95 & 0.32 \\
\hline $\begin{array}{l}\text { Engineering } \\
\text { Services }\end{array}$ & 40 & 4.26 & 0.47 \\
\hline $\begin{array}{l}\text { Hazardous Waste } \\
\text { Management }\end{array}$ & 13 & 3.60 & 0.66 \\
\hline Education Services & 4 & 4.74 & 0.91 \\
\hline $\begin{array}{l}\text { Help Supply } \\
\text { Services }\end{array}$ & 1 & 1.56 & - \\
\hline Oil Services & 23 & 2.90 & 0.42 \\
\hline $\begin{array}{l}\text { Special Industrial } \\
\text { Machinery }\end{array}$ & 52 & 2.47 & 0.15 \\
\hline
\end{tabular}

dard deviation of days of backlog for each company) between 1990 and 2003. This time range was chosen because it covers the period in which companies have been applying more modern supply chain management principles. The 
third column lists the industry average of the number of standard deviations average days of backlog is away from zero. The fourth column lists the standard error of the data in column 3. Note that in almost every industry, these services hold average days of backlog at 2.5 standard deviations or higher away from zero. Thus, it appears that results generated by the linear relaxation models would have efficacy in these industries.

\section{REFERENCES}

Albright, S. C., W. L. Winston, C. Zappe. 2003. Data Analysis and Decision Making with Microsoft Excel, Second Edition. Pacific Grove, CA: Duxbury Press.

Anderson, E.G. and D.J. Morrice. 2002. Capacity and Back$\log$ Management in Queuing-Based Supply Chain. In Proceedings of the 2002 Winter Simulation Conference, ed. E. Yücesan, C. H. Chen, J. L. Snowdon, and J. M. Charnes, 1302-1305. Piscataway, New Jersey: Institute of Electrical and Electronics Engineers.

Anderson, E.G. and D.J. Morrice. 2000. A Simulation Game for Service-Oriented Supply Chain Management Journal of Production and Operations Management 9: 40-55.

Anderson, E.G., D.J. Morrice, and G. Lundeen. 2004a. Stochastic Optimal Control of Staffing and Backlog Policies in a Two-Stage Customized Service Supply Chain. Working paper. The University of Texas at Austin.

Anderson, E.G., D.J. Morrice, and G. Lundeen. 2004b. The "Physics" of Capacity and Backlog Management in Service and Custom Manufacturing Supply Chains. Working paper. The University of Texas at Austin.

Box, G. E. P. and N. R. Draper. 1987. Empirical ModelBuilding and Response Surface. New York, NY: Wiley.

Compustat Industrial Annual Data. 2004. Wharton Research Data Services, http://wrds.wharton.upenn.edu/, The Wharton School, The University of Pennsylvania.

Dejonckheere, J., S. M. Disney, M. R. Lambrecht, and D. R. Towill. 2003. Measuring and Avoiding the Bullwhip Effect: A Control Theoretic Approach. European Journal of Operational Research 147: 567-590.

Kempf, K. G. 2004. Control-Oriented Approaches to Supply Chain Management in Semiconductor Manufacturing. To appear in Proceedings of the 2004 American Control Conference.

Law, A.M., and Kelton, W.D., 2000. Simulation Modeling and Analysis, 3rd Edition. , New York, NY: McGrawHill.

Perea-Lopez, E., B. E. Ydstie, and I. E. Grossman. 2003. A model predictive control strategy for supply chain optimization. Computers and Chemical Engineering 27: 1201-1218.

Perea-Lopez, E., I. E. Grossman, and B. E. Ydstie. 2001. Dynamic Modeling and Decentralized Control of Supply Chains. Industrial \& Engineering Chemistry Research 40(15): 3369-3383.
Towill, D. R. 1996. Industrial Dynamics Modeling of Supply Chains. International Journal of Physical Distribution \& Logistics 26(2): 23-42.

Towill, D. R. 1995. The Application of Filter Theory to the Study of Supply Chain Dynamics. Production Planning and Control 5(1): 82-96.

\section{AUTHOR BIOGRAPHIES}

EDWARD G. ANDERSON is an Associate Professor of Operations Management at the University of Texas McCombs School of Business. He received his doctorate from the Massachusetts Institute of Technology and his bachelor's degree in electrical engineering and history from Stanford University. His research interests include supply chain management, outsourced product development, knowledge management, and system dynamics. He has published articles in such journals as Management Science, Production and Operations Management, and The Systems Thinker. Dr. Anderson won the prestigious Wickham Skinner Early-Career Research Award from the Production and Operations Management Society. He is a senior editor in New Product Development for Production and Operations Management. Professor Anderson has consulted with Ford, Dell, Hewlett-Packard, Frito-Lay, and Atlantic-Richfield. Prior to his academic work, he was a product design engineer at the Ford Motor Company, from which he was granted three U.S. patents. His email address is $<$ Edward. Anderson@mccombs .utexas .edu $>$.

DOUGLAS J. MORRICE is a Professor in the Management Department at The University of Texas at Austin. He has a BA, Honors in Operations Research from Carleton University in Ottawa, Canada. He holds an M.S. and a $\mathrm{Ph} . \mathrm{D}$. in Operations Research and Industrial Engineering from Cornell University. His research interests include operations simulation design, modeling, and analysis, and optimization and risk management in the supply chain. Dr. Morrice is an Area Editor in Simulation Output Analysis for ACM Transactions on Modeling and Computer Simulation and a Senior Editor in Supply Chain Management for Journal of Production and Operations Management. He served as the Secretary for the INFORMS College on Simulation (1994-1996), Co-Editor of the Proceedings of the 1996 Winter Simulation Conference, and Program Chair of the 2003 Winter Simulation Conference. His email address is<morrice@mail.utexas.edu>.

SAURAV BHARADWAJ is a graduate student in the Mechanical Engineering Department at The University of Texas at Austin. He has a BS, in Metallurgical and Materials Engineering from National Institute of Technology in Rourkela, India. He is currently pursuing his M.S. in Manufacturing and Decision Systems. He has two years of information technology experience and has led projects for 
GE-Supply, Cendent Corporation in the area of inventory, revenue and supply chain solutions. He has also worked as a process consultant for Allegiance Telecom. His email address is <sauravb@mail.utexas.edu >. 